\title{
New tubular bioabsorbable knitted airway stent: Biocompatibility and mechanical strength
}

\author{
Yukihito Saito, MDa \\ Kenichirou Minami, MDa \\ Masashi Kobayashi, MDa \\ Yoshihisa Nakao, MDa \\ Hideyasu Omiya, MDa \\ Hiroji Imamura, MDa \\ Noriko Sakaida, MD ${ }^{b}$ \\ Akiharu Okamura, MDc
}

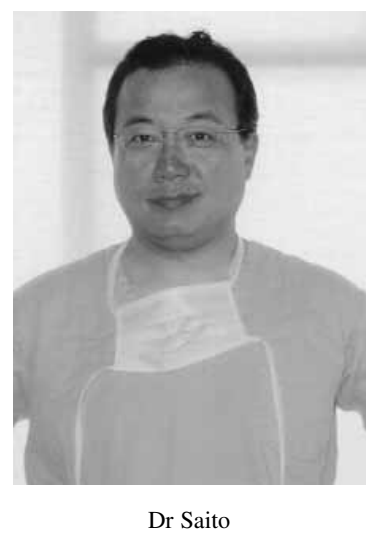

From the Department of Thoracic and Cardiovascular Surgery, ${ }^{\mathrm{a}}$ the Division of Surgical Pathology, ${ }^{\mathrm{b}}$ Kansai Medical University, Moriguchi, and the Division of Surgical Pathology, ${ }^{\mathrm{c}}$ International Center for Diagnostic Pathology, Kobe, Japan.

Received for publication April 3, 2001; revisions requested May 14, 2001; revisions received June 4, 2001; accepted for publication July 3, 2001.

Address for reprints: Yukihito Saito, MD Associate Professor, Department of Thoracic and Cardiovascular Surgery, Kansai Medical University, 10-15 Fumizonocho, Moriguchi, Osaka 570-8507, Japan (E-mail: saitoy@ takii.kmu.ac.jp).

J Thorac Cardiovasc Surg 2002;123:161-7

Copyright (C) 2002 by The American Association for Thoracic Surgery

$0022-5223 / 2002 \$ 35.00+0 \quad \mathbf{1 2 / 1 / 1 1 8 5 0 3}$

doi:10.1067/mtc.2002.118503
Objective: This study examines the biocompatibility and suitability of a new tubular bioabsorbable knitted stent made of poly-L-lactic acid in normal rabbit airways and examines the mechanical strength of this stent in vitro.

Methods: A tubular knitted airway stent (group $\mathrm{B}, \mathrm{n}=15$ ) made of poly-L-lactic acid wire was implanted operatively in New Zealand White rabbits intratracheally; silicone stents served as controls (group $\mathrm{A}, \mathrm{n}=8$ ). The cervical trachea was exposed, and the stent was implanted. Up to 40 weeks after stent implantation, the rabbits were killed, at which time bronchoscopy, histologic examination, and scanning electron microscopic study was done. We tested poly-L-lactic acid stents and silicone stents for their mechanical strength in vitro. We subjected stents to area loads and measured their mechanical strengths.

Results: In group A, which received silicone stents, 3 (37.5\%) rabbits died within 4 weeks of stent implantation as a result of airway obstruction by secretions inside the stent lumen. In group B, poly-L-lactic acid stents, 1 (6.7\%) rabbit died 3 weeks after implantation because of weakness caused by anorexia. In the remaining animals, except for 1 animal with stent trouble, the bronchial lumen was fully open until the 40th week after implantation. After 40 weeks of follow-up, the stents disappeared, except for nonabsorbable suture in the bronchial wall. None of the animals in group B died of airway complication. Histologic examination and scanning electron microscopic examination of the group A silicone stents showed marked regression of ciliated cells under the stent. In group B the ciliated epithelium was preserved, and there were numerous capillary blood vessels in the submucosa. In scanning electron microscopy of the group B polyL-lactic acid stents, the ciliated cells were preserved between the mesh holes of the stent. For diameters between 4 and $6 \mathrm{~mm}$, the mechanical strength of silicone stents was greater than that of poly-L-lactic acid stents. However, the mechanical strength of polyL-lactic acid stents increased as a function of their diameter.

Conclusion: A new tubular bioabsorbable stent made of poly-L-lactic acid is biocompatible in normal rabbit airways, indicating that poly-L-lactic acid is a promising material for airway stents for clinical use.

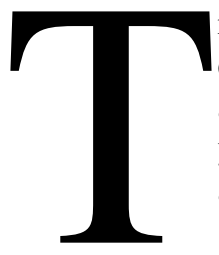

racheobronchial stenoses can be caused by tracheobronchomalacia, extrinsic compression, postintubation tracheal injuries, sequelae after tracheostomy, or malignant or benign tumors. An ideal stent has yet to be developed. The most common types of stents in clinical use are made of silicone or metallic wire. 

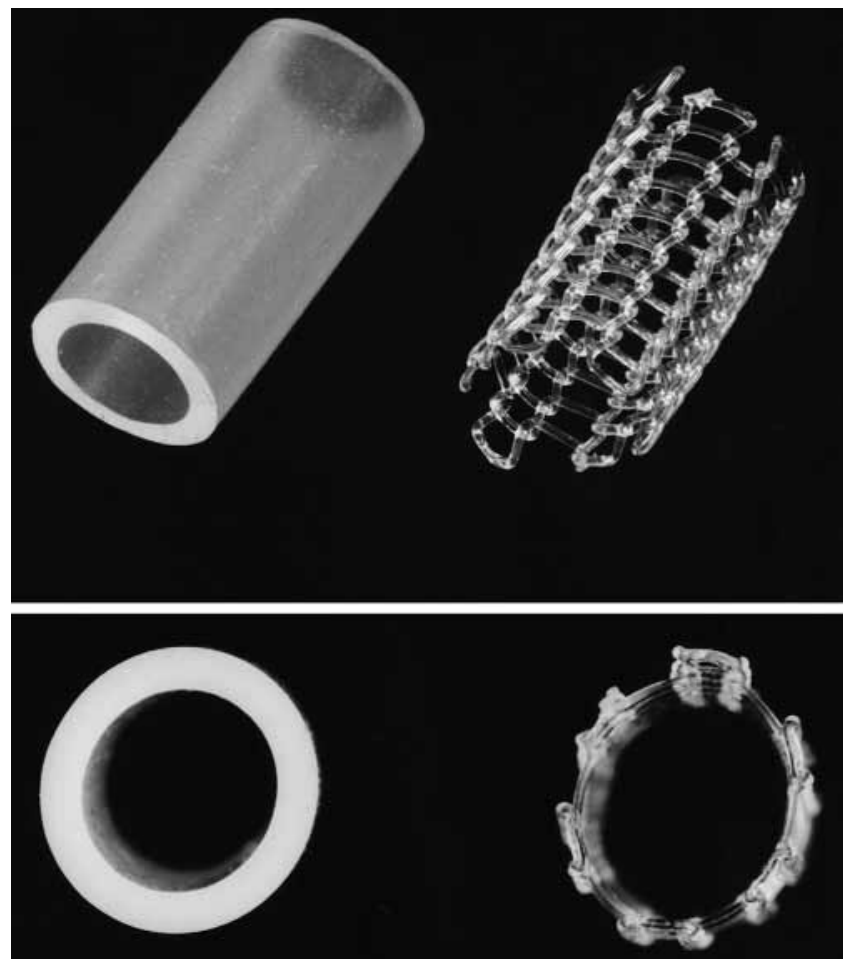

Figure 1. Silicone stent (left) and tubular knitted PLLA stent (right).

The Dumon stent, a silicone tube, has been well established, especially for treatment of stenoses with intraluminal tumor growth or granulomatous tissue. A metallic stent, the nitinol self-expandable airway stent, is usually used in patients with airway stenosis because of extrinsic tumoral compression.

Metallic stents are sometimes used in patients with benign bronchial stenosis after lung transplantation caused by granulation of the bronchial anastomosis site or in pediatric patients with congenital tracheal stenosis and tracheobronchomalacia. Metallic stents have the following advantages compared with silicone stents: (1) simplicity of insertion and fixation; (2) better clearance secretions; (3) accommodation to varying tracheal dimensions; and (4) a low internal/external diameter ratio. However, metallic stents are not without problems. Once covered by epithelium, they are not easy to remove with a conventional bronchoscopic procedure alone, and an operation may be required.

Silicone stents can be removed, but they have several problems, such as disturbance of the physiologic mucociliary function of the tracheobronchial epithelium under the stent. Silicone stents are relatively thick and cause mucociliary function to be lost in the stented area. Secretions tend to accumulate in the lumen, which can cause obstruction.

In the pediatric patient who is growing, it may be necessary to exchange existing stents for larger ones. However,

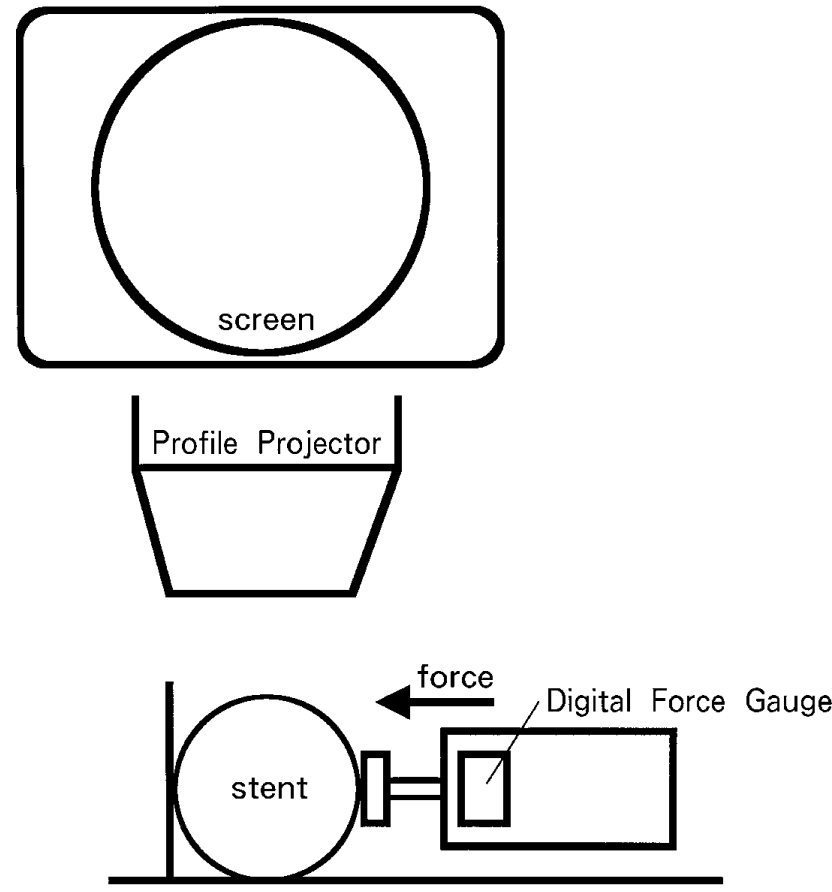

Figure 2. Schema of measurement of diameter of stents with a noncontact profile projector.

this is not easily done if the stent is metallic. Bioabsorbable airway stents, on the other hand, offer benefits: extraction of the device is unnecessary, and normal airway is preserved after stent resorption.

The aim of this study was to examine the biocompatibility and mechanical strength of a new tubular bioabsorbable stent made of poly-L-lactic acid (PLLA).

\section{Materials and Methods}

\section{Evaluation of Biocompatibility in 2 Types of Airway Stents}

The PLLA knitted tubular tracheal stents were made of $170-\mu \mathrm{m}$ wire with an outer diameter of $6 \mathrm{~mm}$ and a length of $12 \mathrm{~mm}$. The silicone stents were tubes $0.8 \mathrm{~mm}$ thick, with an outer diameter of $6 \mathrm{~mm}$ and a length of $12 \mathrm{~mm}$ (Figure 1).

The rabbits (New Zealand White rabbits) were allocated to one of 2 groups. We used silicone stents for control group A, which comprised 8 rabbits with a mean weight of $3.10 \mathrm{~kg}$ (range, 2.70$3.50 \mathrm{~kg}$ ). We used PLLA stents in experimental group B, which comprised 15 rabbits with a mean weight of $3.10 \mathrm{~kg}$ (range, 2.65$3.60 \mathrm{~kg}$ ).

The rabbits were anesthetized with subcutaneous injection of atropine $(0.75 \mathrm{mg})$ and inhalation of $1.5 \%$ halothane. The animals maintained spontaneous breathing without intubation. In the midline the cervical trachea was prepared under direct visualization for implantation of the stent. Unnecessary dissection was avoided so as not to interfere with the blood circulation of the trachea. The trachea was opened transversely between cartilage rings for two 
TABLE 1. Postimplantation course of animals

\begin{tabular}{llcc}
\hline & $\mathbf{8} \mathbf{w k}$ & $\mathbf{2 0} \mathbf{w k}$ & $\mathbf{4 0} \mathbf{w k}$ \\
\hline Silicone $\left(\mathrm{n}=8 ; 3\right.$ died $\left.^{*}\right)$ & 5 (killed) & 0 & 0 \\
PLLA $(\mathrm{n}=15 ; 1$ diedt) & 5 (killed) $\ddagger$ & 5 (killed) & 4 (killed) \\
\hline
\end{tabular}

*Three animals died from airway obstruction within 3 weeks after stent implantation.

tOne animal died of loss of weight with anorexia 3 weeks after implantation. $\ddagger$ Stent trouble (incomplete expansion of the stent) was observed in 1 animal.

thirds of its circumference. The stent was implanted intratracheally and fixed with the same continuous 5-0 polypropylene suture used to close the tracheostomy.

In group A the rabbits were killed at 8 weeks (5 rabbits) after stent implantation. In group B the animals were killed at 8 weeks (5 rabbits), 20 weeks (5 rabbits), and 40 weeks (4 rabbits) after stent implantation, at which time tracheal endoscopy was done. The cervical trachea was excised and divided longitudinally into two pieces, one fixed in formalin solution for histologic examination and the other in buffered glutaraldehyde solution for scanning electron microscopic study. All of the surgical and euthanasia procedures were performed in accordance with the "Guide for the Care and Use of Laboratory Animals" prepared by the Institute of Laboratory Animal Resources, National Research Council, and published by the National Academy Press, revised 1996.

\section{In Vitro Evaluation of Mechanical Strength in 2 Types of Airway Stents}

We tested PLLA stents and silicone stents in vitro for their mechanical strength. Using a digital force gauge (DFG-0.2K; Shimpo Corp, Kyoto, Japan) and a noncontact profile projector (PJ 300; Mitsutoyo Corp, Kyoto, Japan), we subjected stents to area loads at room temperature $\left(22^{\circ}-24^{\circ} \mathrm{C}\right)$ and measured their deformation rates. Area load was applied over the full length of the stent with a $50-\mathrm{mm}^{2}$ plate mounted on a digital force gauge with a measuring range of $\pm 1.960 \mathrm{~N}$ and an accuracy of $\pm 1 \mathrm{mN}$. The stent diameter before and after loading was measured 6 times each on the screen of a noncontact profile projector to an accuracy of 1/1000 $\mathrm{mm}$ (Figure 2). We calculated the deformation rate as follows:

Deformation rate $(\%)=$

$$
\frac{\text { Diameter before loading }- \text { diameter after loading }}{\text { Diameter before loading }} \times 100 \text {. }
$$

\section{Results \\ Evaluation of Biocompatibility in 2 Types of Airway Stents}

All rabbits tolerated the surgical operation and the implantation of the stent. Perioperative complications included airway obstruction and, in the neck of 1 rabbit, a subcutaneous abscess.

In group A, which received silicone stents, 3 rabbits died within 4 weeks of stent implantation as a result of airway obstruction by secretions inside the stent lumen. The 4-
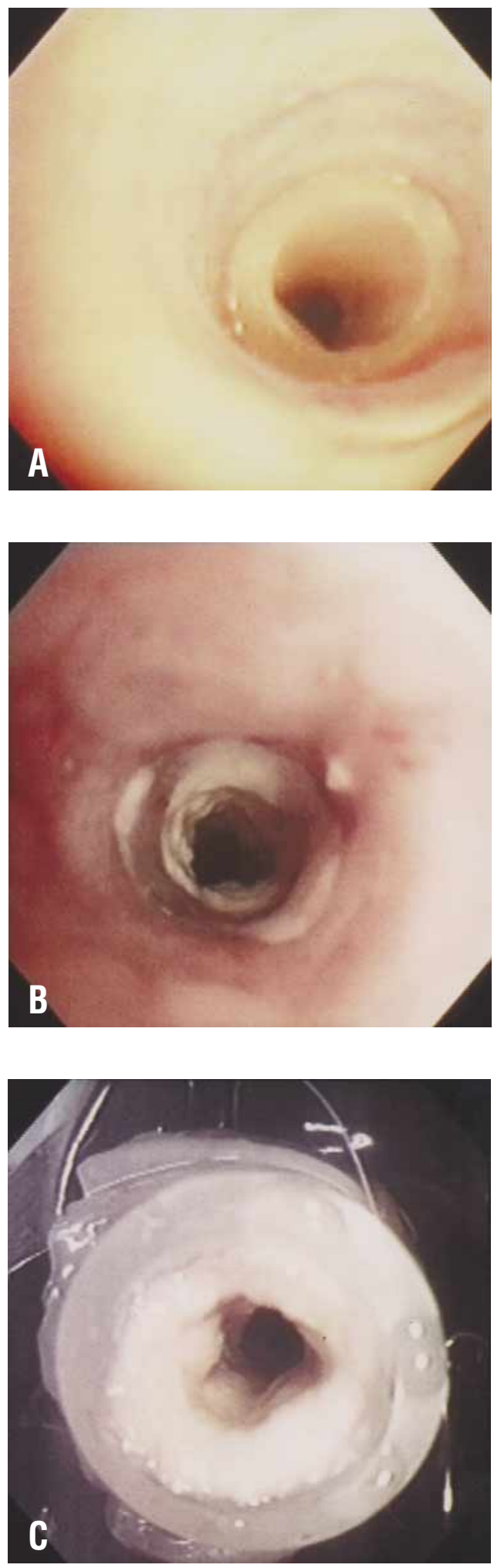

Figure 3. Bronchoscopic findings in group $A$ (silicone): $A$, Immediately after stent implantation; B, severe airway stenosis 8 weeks after implantation; and $C$, after removal of silicone stent, obstructive material was visible inside the stent. 

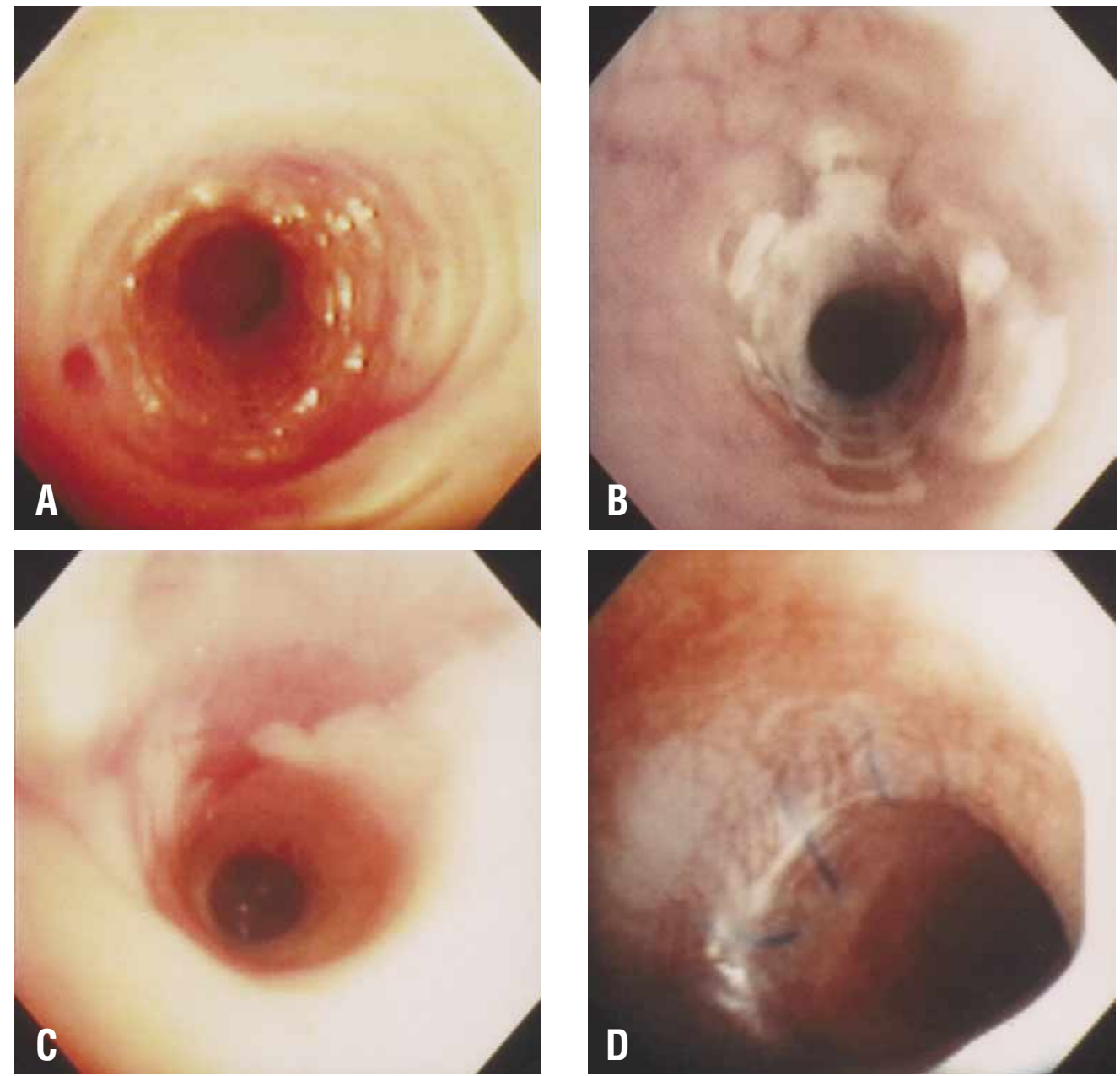

Figure 4. Bronchoscopic findings in group B (PLLA). A, Immediately after stent implantation; B, 8 weeks after implantation, airway was fully open; $C, 20$ weeks after implantation, a part of the PLLA stent was not visible because it was covered with tracheal epithelium; and D, 40 weeks after implantation, the PLLA stent was absorbed, and nonabsorbable suture in the bronchial wall was observed.
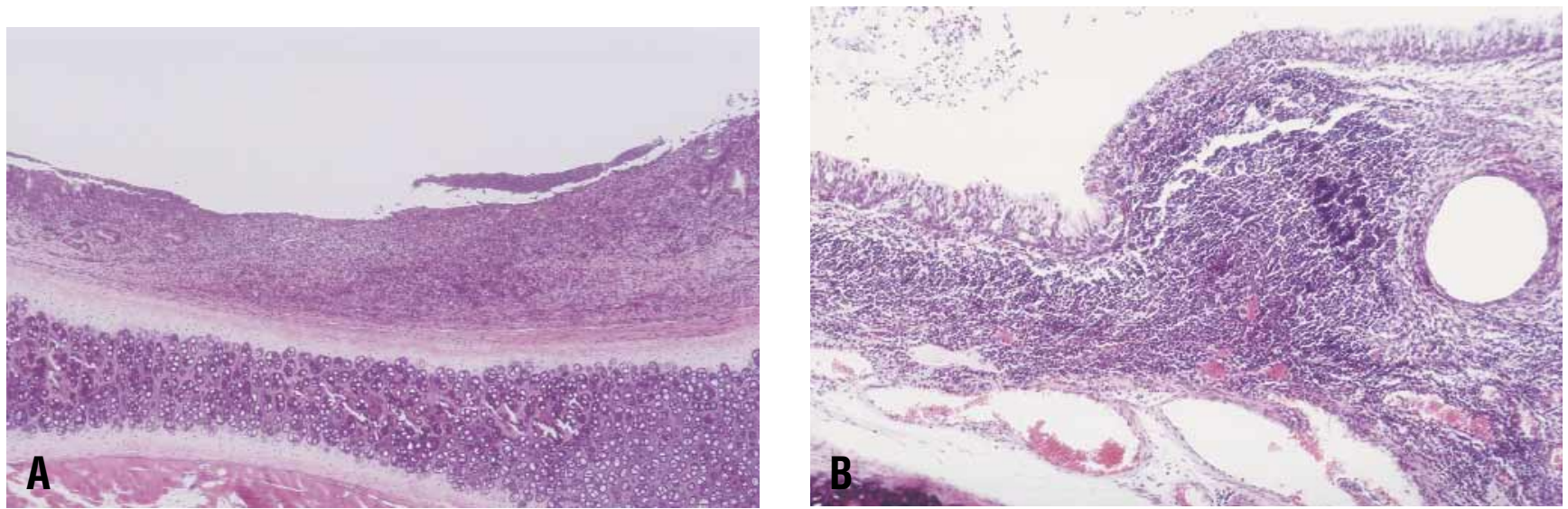

Figure 5. Histologic findings in both groups. A, Epithelial erosion was observed under the stent 8 weeks after implantation (group A). B, Epithelium was preserved, and numerous capillary blood vessels in the submucosa were observed 8 weeks after implantation (group B).

164 The Journal of Thoracic and Cardiovascular Surgery • January 2002 

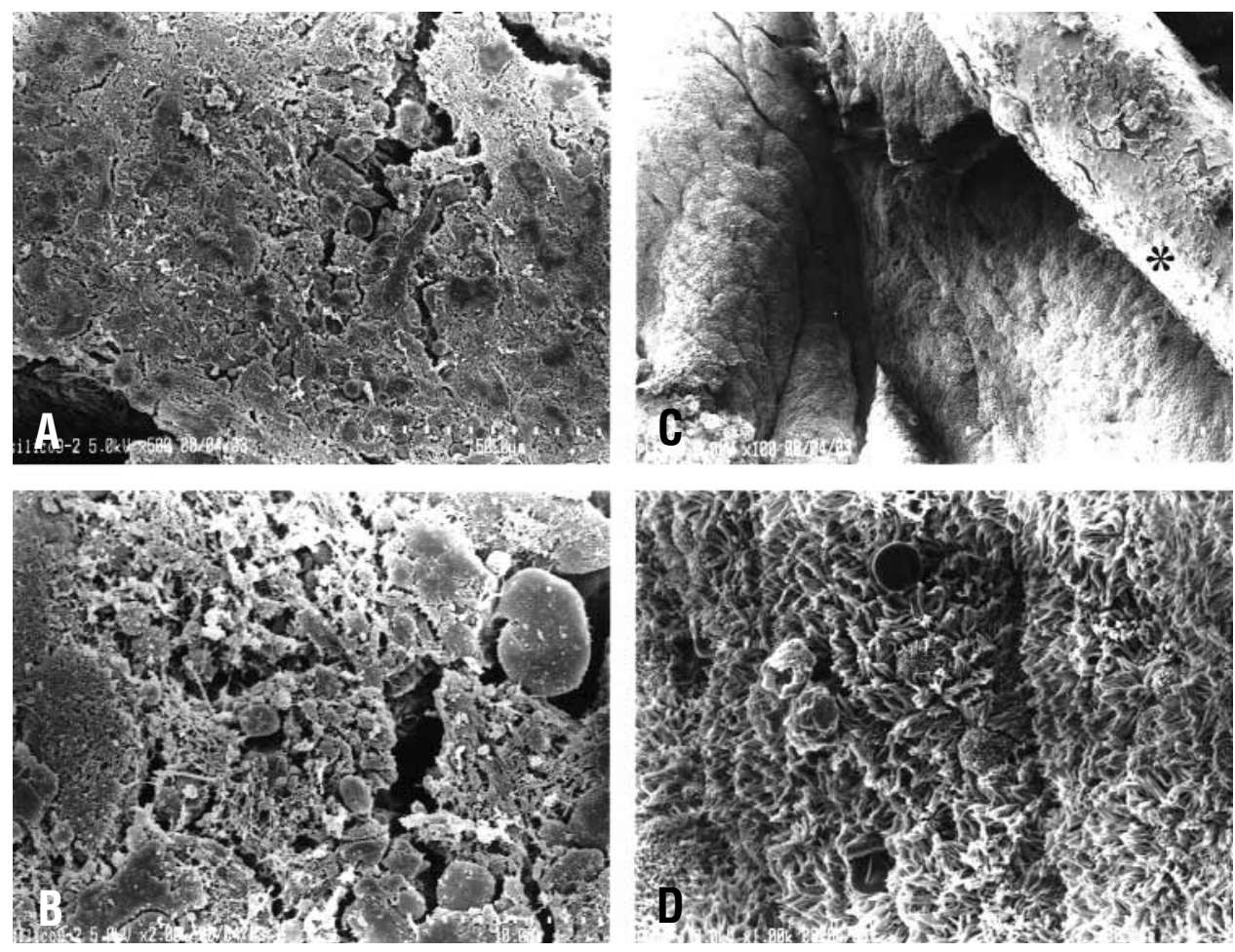

Figure 6. Scanning electron microscopic findings in both groups. A and B, Marked regression of ciliated cells under the stent 8 weeks after implantation (group A). (A, original magnification 500x; B, original magnification 2000x.) C and D, Ciliated cells were preserved between the mesh holes of the stent 8 weeks after implantation (group B). (C, original magnification 100x; D, original magnification 1000x.) *PLLA wire.

week survival of the rabbits in group A was $62.5 \%$. In the remaining rabbits stridor developed within 8 weeks after stent implantation. Therefore, all rabbits surviving up to 8 weeks after stenting were killed in the eighth postoperative week. Obstructing material was visible inside the stents of all rabbits (Figure 3 ).

In group B, which received PLLA stents, only 1 animal died 3 weeks after implantation because of weakness caused by anorexia. At autopsy, this animal was found to have a subcutaneous abscess of the neck, but the bronchial lumen was not stenosed. The 4-week survival of the rabbits in group A was $93.3 \%$. In the remaining animals, except for 1 animal with stent trouble (incomplete expansion of the knitted wire), the bronchial lumen was fully open until the 40th week after stent implantation (Table 1). After 40 weeks' follow-up, the stents disappeared, except for nonabsorbable sutures in the bronchial wall (Figure 4). None of rabbits in group B died of airway complications.

Histologic examination of the group A silicone stents at 8 weeks after implantation showed marked regression of ciliated cells under the stent. In some areas the epithelium had eroded, and there was chronic lymphocytic inflamation in the submucosa (Figure 5, A). There was mucosa swelling at both ends of the stents. In the group B PLLA stents the ciliated epithelium was preserved, and there were numerous capillary blood vessels in the submucosa at 8 weeks after implantation (Figure 5, B). Scanning electron microscopy of the group A silicone stents at 8 weeks after implantation showed marked regression of ciliated cells under the stent (Figure 6, $A$ and $B$ ).

In the group B PLLA stents the ciliated cells were preserved between the mesh holes of the stent 8 weeks after implantation (Figure 6, $C$ and $D$ ).

\section{In Vitro Evaluation of Mechanical Strength in 2 Types of Airway Stents}

For diameters between 4 and $6 \mathrm{~mm}$, the deformation rate of the silicone stent was lower than that of the PLLA stent. However, the mechanical strength of PLLA stents increased as a function of their diameter. At a diameter of $8 \mathrm{~mm}$, the deformation rate of PLLA stents was similar to that of silicone stents. At diameters of 10 and $13 \mathrm{~mm}$, the deformation rate of PLLA stents was significantly lower than that of silicone stents (Figure 7).

\section{Discussion}

After the introduction of the Montgomery ${ }^{1}$ T-tube in 1965, one of the most widely used types of silicone stents was 


\section{deformation rate}
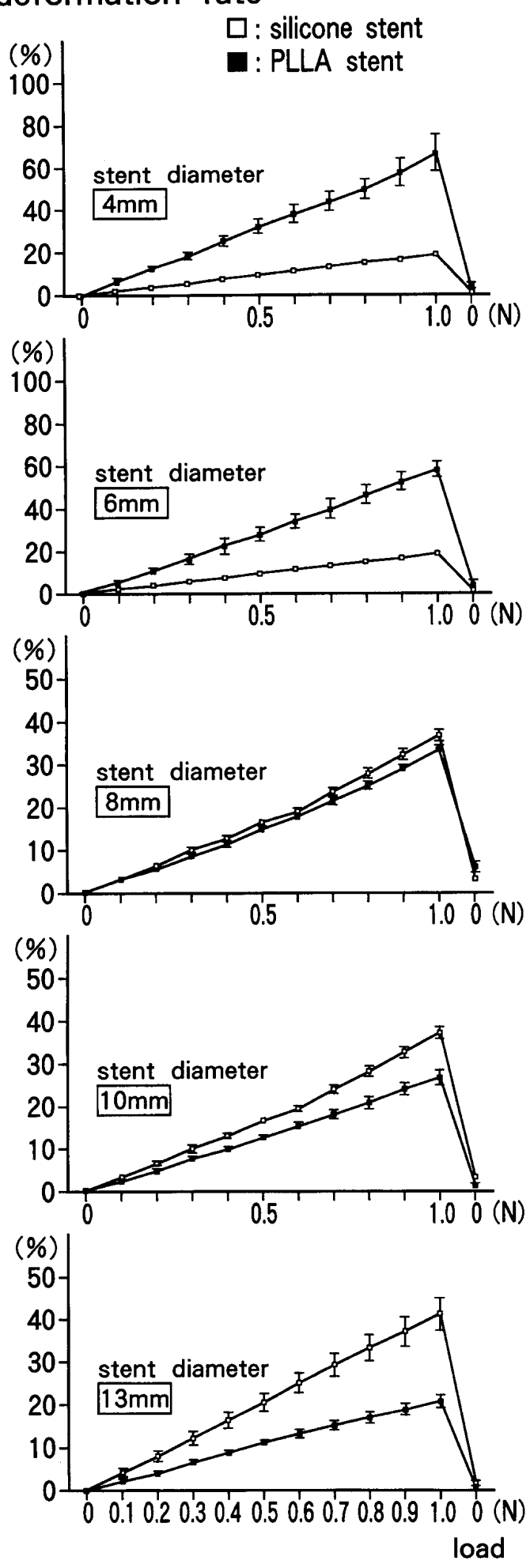

Figure 7. Comparison of deformation rate in 2 types of airway stents. Between 4 and $6 \mathrm{~mm}$, the deformation rate of silicone stents was significantly lower than that of PLLA stents. At diameters of 10 and $13 \mathrm{~mm}$, the deformation rate of PLLA stents was significantly lower than that of silicone stents. designed by Dumon. ${ }^{2}$ Because silicone stents are relatively thick, the internal diameter of the stent is thus smaller than the normal airway lumen. The stents interfere with normal airway mucociliary function, which can result in accumulation of secretions inside the stent and obstruction of its lumen.

The ideal stent should be simple to insert, fix, and remove; be biocompatible; not obstruct the airway; allow clearance of secretions; and accommodate to varying tracheal dimensions and shapes. ${ }^{3,4}$

Metallic expandable wire stents, such as the Wallstent, ${ }^{5}$ have a low internal/external diameter ratio. They cannot usually migrate after insertion, and the mucociliary clearance function is better maintained. However, once covered by epithelium, it is not easy to remove them with a bronchoscopic procedure. An operation may be required.

Silicone and metallic stents used in the tracheobronchial tree for the treatment of airway problems both have disadvantages, as mentioned above. Long-term results in tracheobronchial airway stenting after implantation has not fully been discussed ${ }^{6}$ because airway stents are usually only used for short-term maintenance of quality of life in patients who have advanced malignant airway diseases, mainly caused by lung cancer.

In the pediatric patient who is growing, it may be necessary to exchange existing stents for larger ones. However, this is not easily done if the stent is metallic. Although silicone stent can be exchanged, it is unfavorable for stenting the small airway in a child because of its thick wall. Its most disadvantageous feature is its disturbance of the physiologic mucociliary function of the small airway.

The new tubular bioabsorbable knitted airway stent has advantages, such as a low internal/external diameter ratio and good clearance secretions. Bioabsorbable stents are optimal if the need for stenting the airway is only temporary. For benign and post-transplantation stenosis in particular, a bioabsorbable stent can be an alternative means of treatment.

The biocompatibility of bioabsorbable materials, such as PLLA and polyglycolic acid, has been good in other organs. Rods of bioabsorbable PLLA and pledgets of polyglycolic acid are in clinical use for the fixation of bone fractures and reinforcement of suture lines in lung parenchyma, with favorable results. ${ }^{7-9}$ In our study PLLA was chosen for an absorbable bronchial stent because it has the longest biodegradation time of the basic molecules available. Its biodegradation time is about 6 months. The bioabsorbable PLLA stent examined in our study is a different type from the spiral stent previously reported. ${ }^{10,11}$

The new type of PLLA stents are mechanically strong enough to retain their tubular shape against extrinsic mechanical force. The mechanical strength of the PLLA stent increased as a function of diameter. At diameters of 4 and $6 \mathrm{~mm}$, the PLLA stents we tested were mechanically weaker than the silicone stents; however, knitted tubular 
stents made of PLLA wire thicker than $170 \mu \mathrm{m}$ can be as strong as silicone stents. At diameters of $10 \mathrm{~mm}$ or more, the mechanical strength of PLLA stents was stronger than that of silicone stents. Therefore, for patients with various degrees of airway diseases, knitted tubular PLLA stents can be used like silicone stents.

In conclusion, the biocompatibility of airway stents made of PLLA is good enough to make it a promising material for airway stents in clinical use. This bioabsorbable airway stent can be useful in the treatment of tracheomalacia in a child who is growing.

This bioabsorbable knitted tubular stent and its construction require further investigation before clinical applications begin.

\section{References}

1. Montgomery WW. T-tube tracheal stent. Arch Otolaryngol. 1965;82: 320-1.

2. Dumon JF. A dedicated tracheobronchial stent. Chest. 1990;97:34832.
3. Loeff DS, Filler RM, Gorenstein A, Ein S, Philippart A, Bahoric A, et al. A new intratracheal stent for tracheobronchial reconstruction: experimental and clinical studies. J Pediatr Surg. 1988;23:1173-7.

4. Becker HD. Stenting of central airways. J Bronchology. 1995;2:98106.

5. Bolliger CT, Heitz M, Hauser R, Probst A, Perruchoud AP. An airway Wallstent for the treatment of tracheobronchial malignancies. Thorax. 1996;51:1127-9.

6. Mehta AC, Dasgupta A. Airway stents. Clin Chest Med. 1999;20:13951.

7. Majola A, Vainionpaa S, Vihtonen K. Absorption, biocompatibility and fixation properties of polylactic acid in bone tissue: an experimental study in rats. Clin Orthop. 1991;268:260-9.

8. Hirata T, Fukuse T, Mizuno S, Wada H. Clinical application of biodegradable rib connecting pins in thoracotomy. Thorac Cardiovasc Surg. 1999;47:183-7.

9. Nakamura T, Shimizu Y, Watanabe S, Hitomi S, Kitano M, Matsunobe S. A new bioabsorbable pledgets and non-woven fabrics made from polyglycolide (PGA) for pulmonary surgery. Thorac Cardiovasc Surg. 1990;38:81-5.

10. Korpela A, Aarnio P, Sariola H, Tormala P, Harjula A. Comparison of tissue reactions in the tracheal mucosa surrounding a bioabsorbable and silicone airway stents. Ann Thorac Surg. 1998;66:1772-6.

11. Korpela A, Aarnio P, Sariola H, Tormala P, Harjula A. Bioabsorbable self-reinforced poly-L-lactide, metaric, and silicone stents in the management of experimental tracheal stenosis. Chest. 1999;115:490-5.

\section{Authoritative}

The Journal of Thoracic and Cardiovascular Surgery is the most frequently cited thoracic/cardiovascular surgery journal in the Science Citation Index. An article in JTCVS is sited on average almost twice as often as those in the closest cardiothoracic journal. 